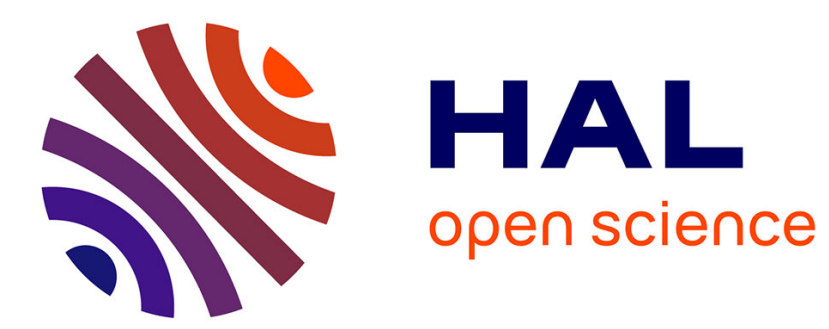

\title{
Curieuses sculptures khmères
}

Bertrand Porte

\section{To cite this version:}

Bertrand Porte. Curieuses sculptures khmères. Arts Asiatiques, 2004, 59, pp.173 - 177. 10.3406/arasi.2004.1526 . halshs-01885966

\section{HAL Id: halshs-01885966 \\ https://shs.hal.science/halshs-01885966}

Submitted on 10 Oct 2018

HAL is a multi-disciplinary open access archive for the deposit and dissemination of scientific research documents, whether they are published or not. The documents may come from teaching and research institutions in France or abroad, or from public or private research centers.
L'archive ouverte pluridisciplinaire HAL, est destinée au dépôt et à la diffusion de documents scientifiques de niveau recherche, publiés ou non, émanant des établissements d'enseignement et de recherche français ou étrangers, des laboratoires publics ou privés. 


\section{Curieuses sculptures khmères}

\section{Bertrand Porte}

\section{Citer ce document / Cite this document :}

Porte Bertrand. Curieuses sculptures khmères. In: Arts asiatiques, tome 59, 2004. pp. 173-177;

doi : https://doi.org/10.3406/arasi.2004.1526

https://www.persee.fr/doc/arasi_0004-3958_2004_num_59_1_1526

Fichier pdf généré le 21/04/2018 


\section{Curieuses sculptures khmères}

Au mois de décembre 2000, un collectionneur américain a fait remettre au Musée national de Phnom Penh, une sculpture masculine sans bras ni jambe (fig. 2), sans fournir aucune documentation sur son origine. Au moment de sa réception par le musée, cette statue, fixée sur un socle métallique, fut exposée, puis retirée des galeries après quelques jours seulement, car sa tête, qui paraissait singulière, maladroite et mal proportionnée, ne correspondait pas au corps sculpté. Les personnels de l'atelier de restauration et moi-même avons été chargés de son examen.

Nous avons détecté aussitôt la présence d'une broche métallique à l'intérieur du cou ainsi qu'un raccord habilement dissimulé. Il était évident qu'une tête, peut-être

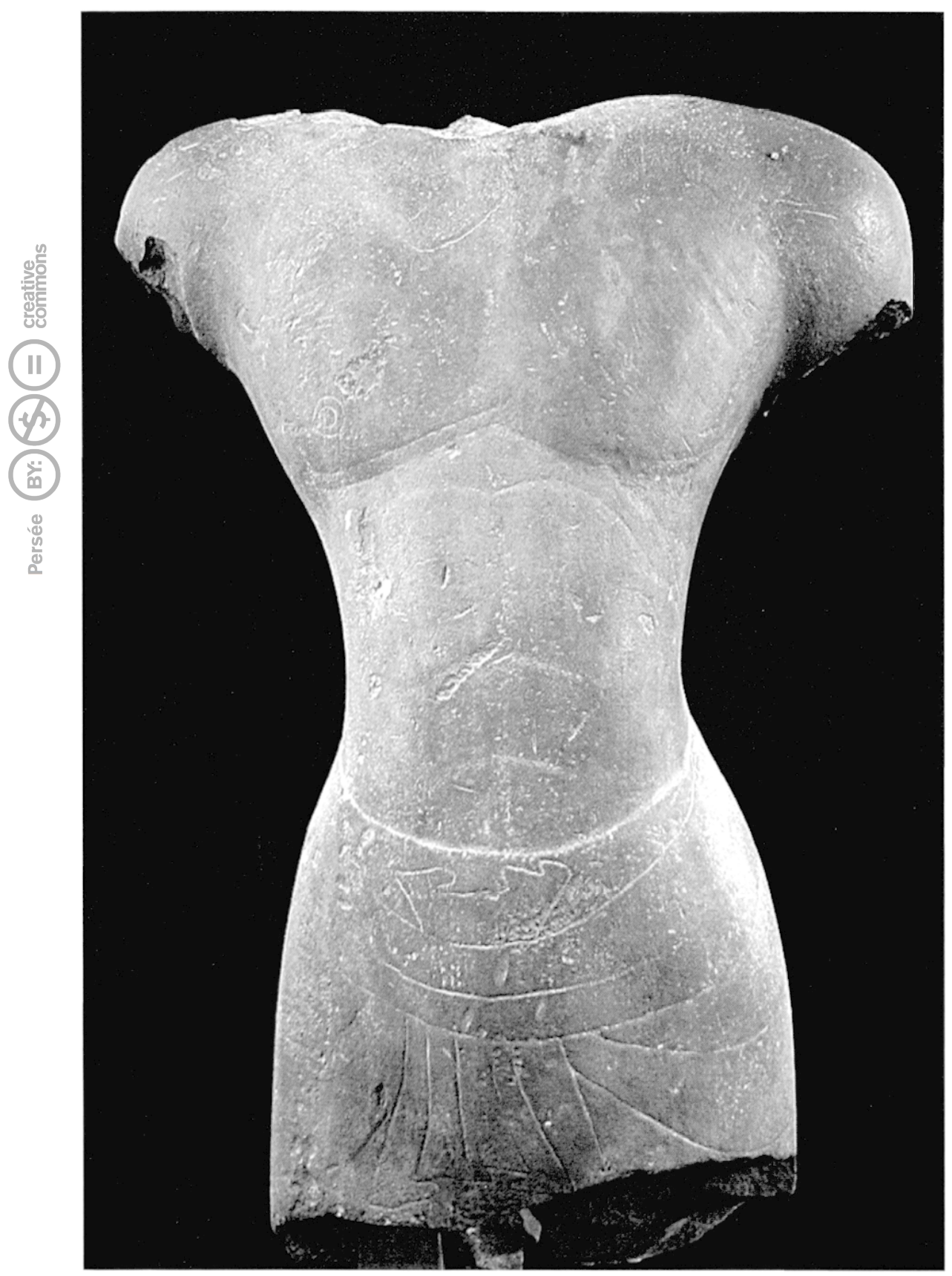

fausse en raison de ses proportions inhabituelles, avait été greffée sur le corps de la statue, à propos duquel aucun soupçon n'avait lieu d'exister au premier regard. La dépose de la tête a cependant apporté une série de surprises. Une couche d'aspect minéral moins dense, brunâtre et relativement épaisse, recouvrait les plans de cassure d'une roche gris bleutée, très fine et dense. Les cassures dégagées ne s'accordaient pas, mais la roche constituant le coeur du tronc et de la tête était bien identique.

Nous avons sondé la nuque de la statue cherchant à mettre en évidence les bords des retouches qui, classiquement pour mieux dissimuler la jointure, débordent et vont «en mourant». Curieusement, nos sondages ne trouvaient pas de limite. Très intrigués, nous avons sondé d'autres zones, et toujours nous retrouvions cette même couche d'épaisseur assez régulière qui habillait la totalité de l'épiderme (fig. 1b). Sous cette couche, la forme et certains détails semblaient déjà ébauchés dans la

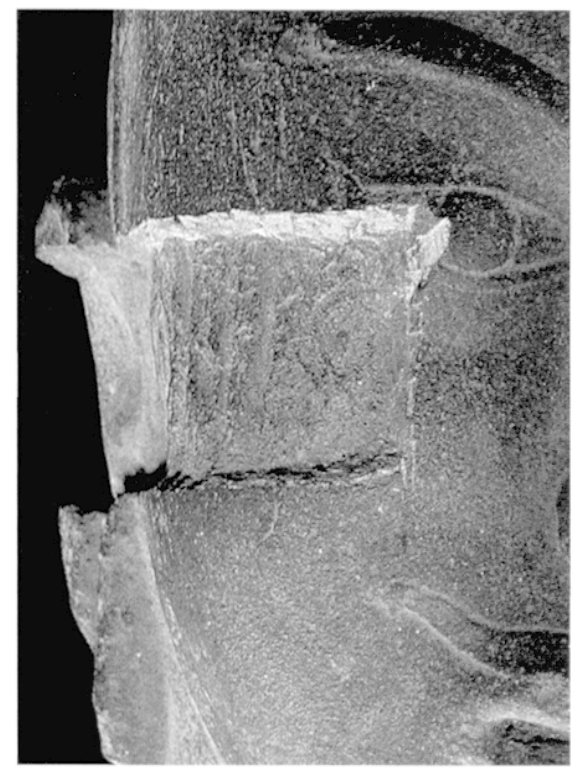

Fig. 1b

Tête, coupe de la couche épidermique (cf. fig. 2)

Fig. 1a

Corps (cf. fig. 2) 


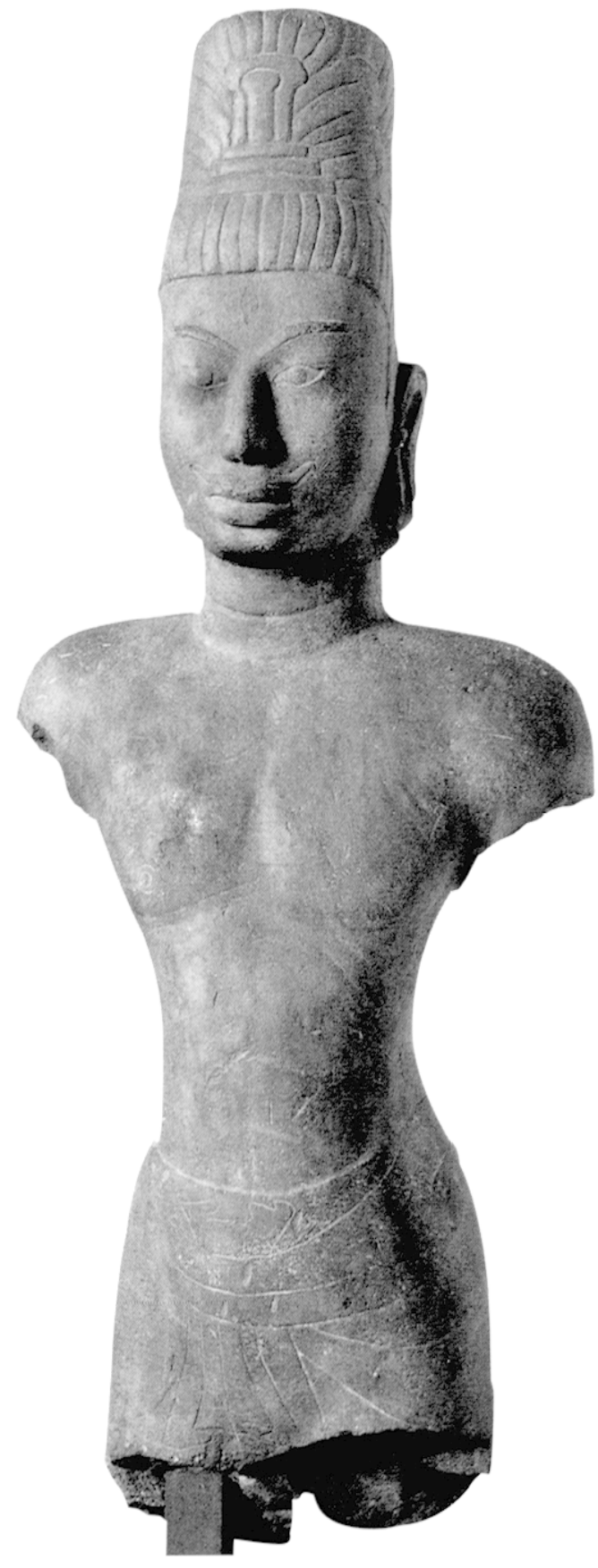

Fig. 2

Corps masculin (tête et corps assemblés: H. $117 \mathrm{~cm} ; \mathrm{L} . .52 \mathrm{~cm} ; \mathrm{P} .25 \mathrm{~cm}$ ). Grès gris bleu à grain fin. Provenance inconnue,

Musée national de Phnom Penh (phot. Atelier du Musée de Phnom Penh)

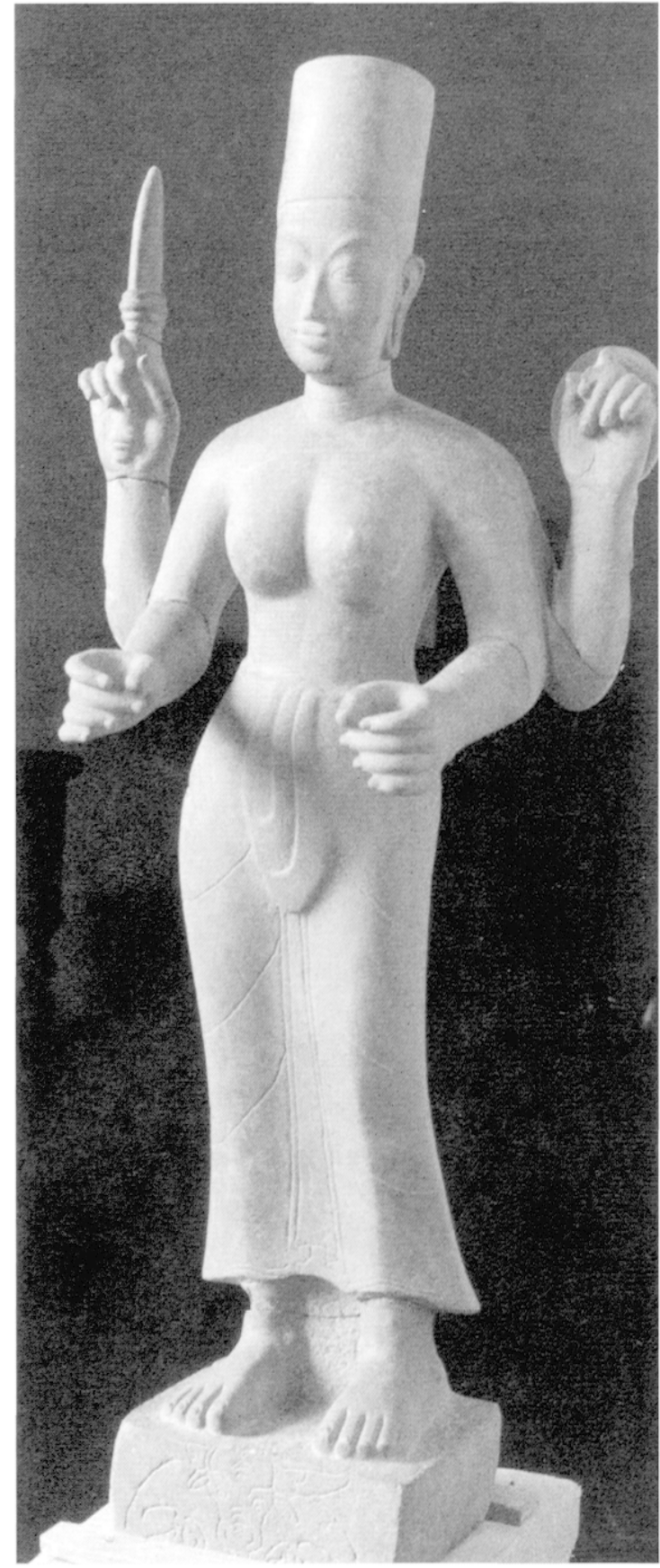

Fig. 3a

Durgā Mahișāsuramardinī (H. $89 \mathrm{~cm} ; \mathrm{L.} 43 \mathrm{~cm} ;$ P. $18 \mathrm{~cm}$ ).

Grès gris à grain fin. Tumlap, province de Kompong Speu, Musée national de Phnom Penh. Ka 2927 (phot. Atelier du Musée de Phnom Penh) pierre, mais des traces de façonnage rappelant celles d'un disque diamanté nous ont conduits par ailleurs à penser que la facture de toutes les parties de cette statue était récente. On pouvait alors penser que cette statue était un faux, que le corps et la tête avaient été sculptés séparément, puis joints ensemble et recouverts enfin d'une couche de mortier teinté afin d'affiner les formes et de donner une patine.
Pour affiner notre étude, nous avons prélevé des échantillons pour analyse. Janet Douglas, du département de conservation et de recherche scientifique de la Smithsonian Institution (Freer Gallery of Art et Arthur M. Sackler Gallery), a bien voulu effectuer ces travaux d'analyse. Les résultats qu'elle a obtenus ont révélé que la roche utilisée dans le cœur de la statue est de nature détritique, et s'apparente à un grès à ciment calcaire de très faible porosité. La couche épidermique brune ne contient pas de ciment calcaire et elle présente une porosité plus marquée. Elle n'est vraisemblablement pas constituée d'un mortier, mais résulte plutôt d'une altération artificielle de la roche par un ou plusieurs bains d'acide destinés à détruire le ciment calcaire du grès, afin de lui conférer après refaçonnage un aspect de surface 


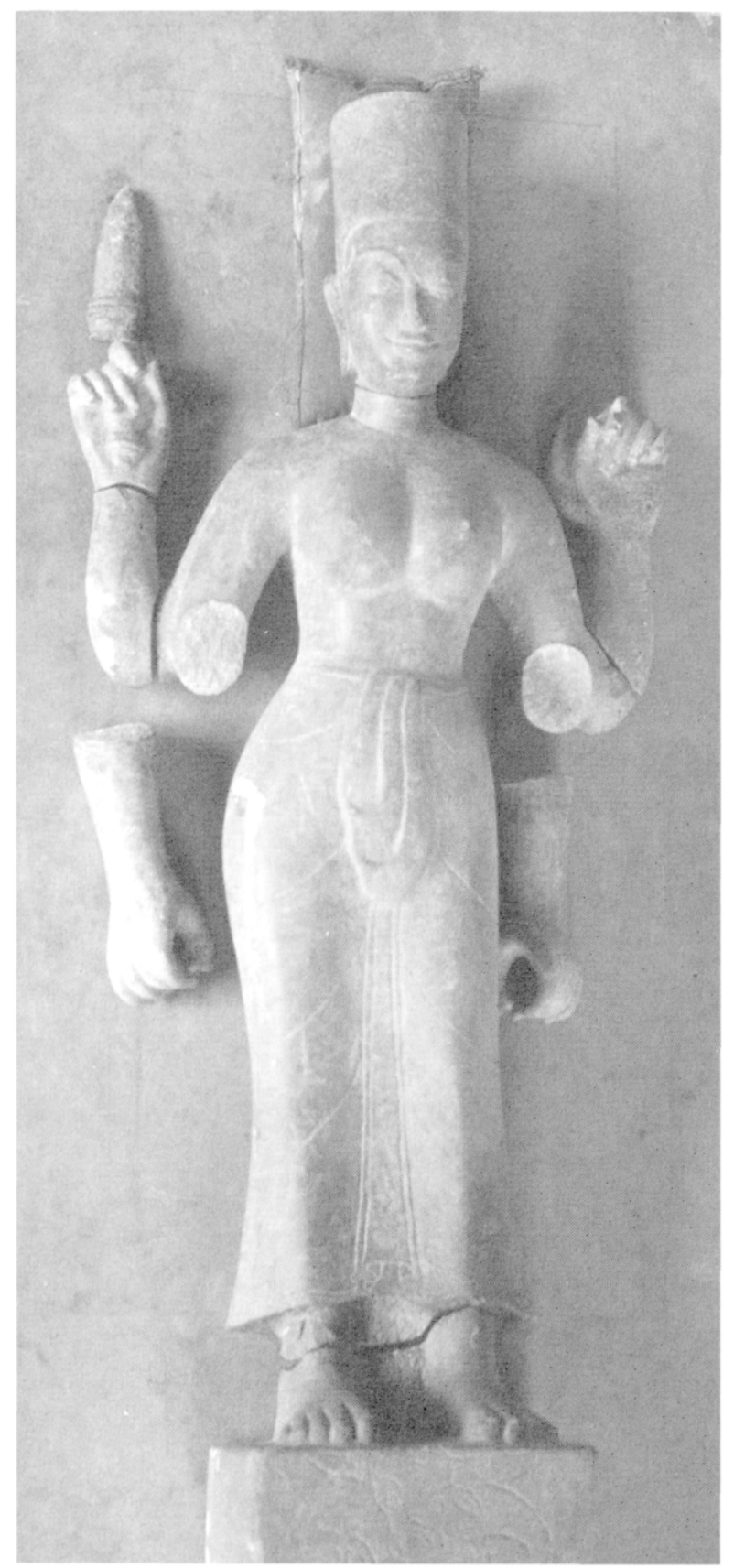

Fig 3b

Durgā Mahişāsuramardinī, en cours de restauration,

Musée national de Phnom Penh, Ka 2927

(phot. Atelier du Musée de Phnom Penh)

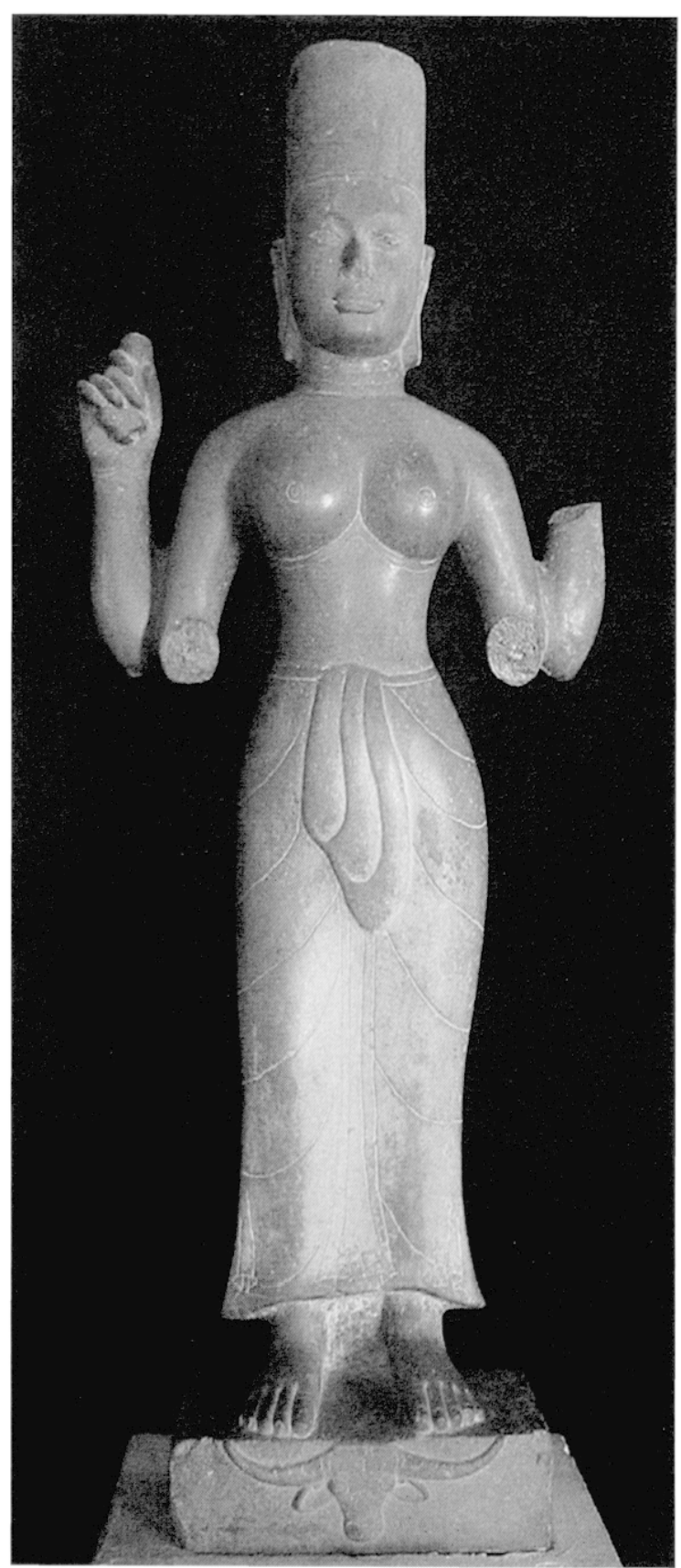

Fig. 4

Durgã (H. $106 \mathrm{~cm})$. Grès.

Tuol Komnat, province de Prey Veng, style de Prei kmeing. fin vil'-début vill's., Musée national de Phnom Penh, Ka 1631 (phot. Atelier du Musée de Phnom Penh) ressemblant à la «patine» naturelle du grès.

Si la tête ajoutée à la statue n'avait pas été si maladroite dans sa facture, il est probable que son corps, beaucoup mieux imité (fig. 1a), serait encore aujourd'hui exposé dans les galeries du musée. Nous sommes donc en présence d'un faux, exécuté avec des procédés sophistiqués pour le rendu des matières et qui, certainement, résul- tent de travaux d'approche méthodiques. Aujourd'hui seulement, avec le recul qui est le nôtre et instruits par les examens que nous avons effectués, l'imitation nous apparaît flagrante.

Une Durgā Mahișāsuramardinī (fig. 3a), arrivée en 2002 au musée de Phnom Penh et publiée dans un ouvrage récent', ayant été confiée à nos soins, a attiré notre attention de la même manière. L'ceuvre, bien que cassée en neuf morceaux, était exceptionnellement bien conservée: il ne lui manquait qu'un seul doigt et elle portait seulement une profonde éraflure due, selon les informations dont nous disposions, à un "coup de pioche" sur le flanc. Les plans de cassure laissaient apparaître "le grès à vif», cette pièce n'ayant pas de patine, et tout laissait penser que ces accidents étaient récents. Les circonstances de sa 


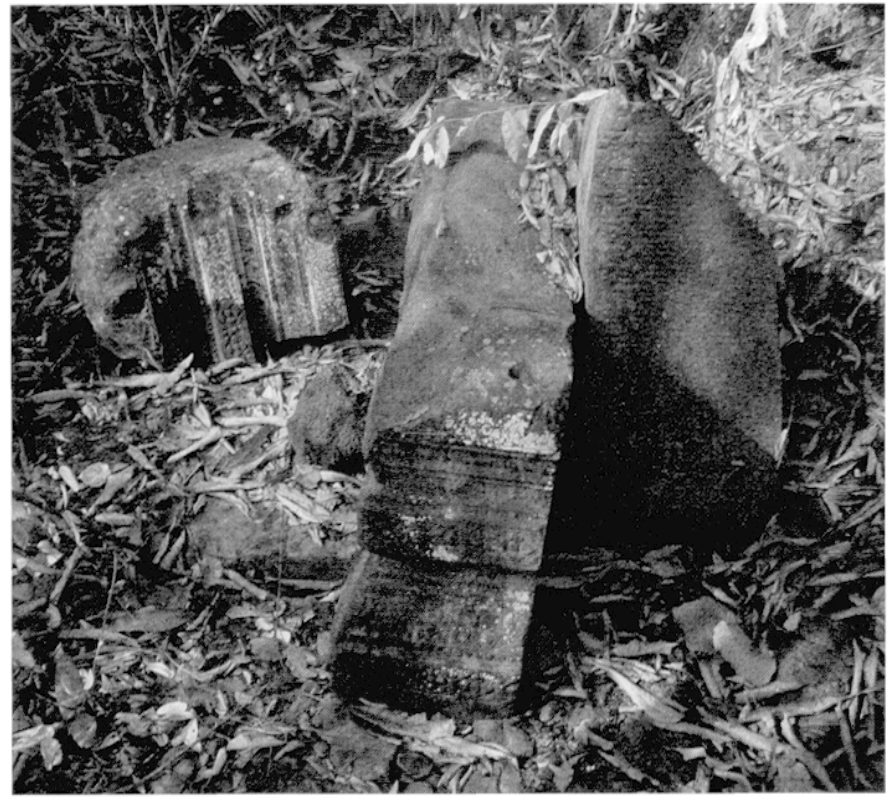

Ce trouble, ajouté au sentiment que nous avions eu lors de la restauration de la pièce dont les joints cassure étaient si parfaits, comme tout récents, nous permettait de mettre en doute l'authenticité de cette sculpture.

Nous terminerons cet exposé de nos récentes investigations par le cas plus complexe d'une œuvre publiée dans le récent ouvrage que nous avons mentionné précédemment ${ }^{3}$. Il s'agit d'un Ganeśa monumental $(165 \times 130 \times 85 \mathrm{~cm})$ du style de Koh Ker, dont la provenance n'est pas précisée, et qui est publié pour la première fois. Hormis la disparition de ses deux mains, la pièce semble exceptionnellement bien conservée. Comme le notent judicieusement les auteurs, cette statue est comparable au Ganeśa du Prasat Bak de Koh Ker qui a aujourd'hui disparu, et dont il ne reste plus

découverte demeurent obscures. Elle aurait été exhumée deux ans avant son arrivée au musée, lors du creusement d'un bassin à quelques kilomètres au sud de Kompong Speu, sans qu'aucun vestige n'ait été repéré à proximité.

La restauration menée par l'atelier du musée (fig. $3 \mathrm{~b}$ ) a permis de retrouver très précisément l'homogénéité de la pièce, dont les fragments se positionnaient parfaitement sans qu'aucune épaufrure ne gêne les raccords. En restituant l'intégrité de la statue, la restauration permettait, pour la première fois, d'appréhender l'image quasi complète d'une Durgã khmère.

Le caractère exceptionnel de cette pièce a cependant été remis en question lorsqu'elle a été exposée au musée, à côté d'une autre image de même époque et de mêmes proportions, la Durgā de Tuol Komnap² (fig. 4). La comparaison faisait progressivement naître une étrange impression. La Durgā de Tuol Komnap apparaissait comme le modèle qui aurait fortement inspiré sa voisine, mais comme un modèle mal compris: le hanchement, qui balance de l'autre coté, excessivement marqué, presque ouIrancier; les pieds plus lourds; le démon buffle traité seulement par une incision. Ce fut comme si un voile tombait soudainement, et la nouvelle ronde-bosse, pourtant bien exécutée, apparut de facture "plate».

Fig. 5b Gaṇeśa, aujourd'hui disparu (H. avec la base: $251 \mathrm{~cm}$ ). Grès, $X^{r}$ s. Prasat Bac, Koh Ker, 1934 (phot. Mission Henri Parmentier)

Fig. $5 a$

Fragments de la base de la statue de Ganesa. janvier 2004 (phot. C. Pottier, EFEO)

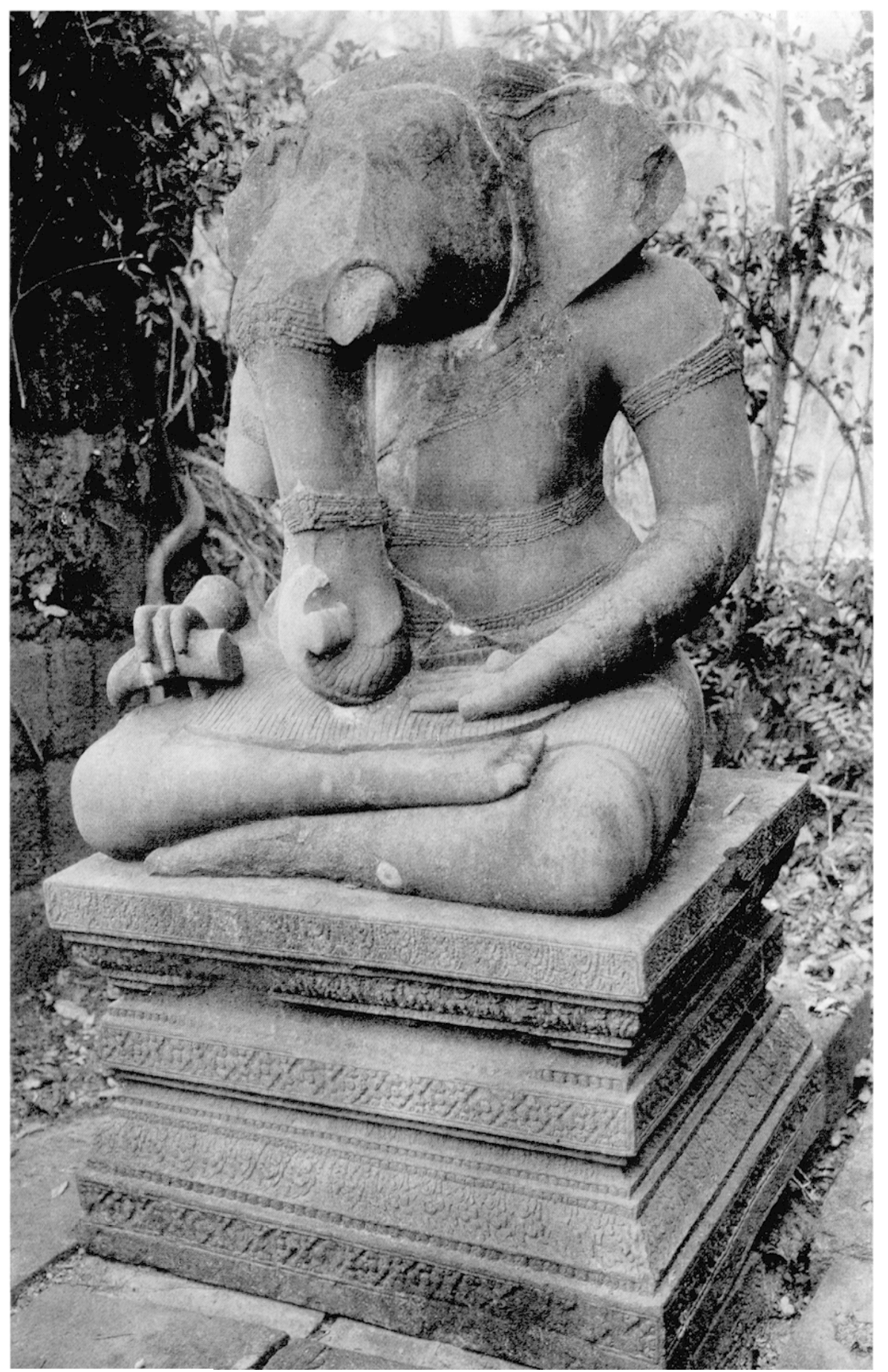


sur le site que trois fragments de la base ${ }^{4}$ (fig. 5a).

Au début des années 1930, Henri Parmentier avait retrouvé la statue ${ }^{5}$ dont les fragments jonchaient l'entrée du temple déjà très ruiné. Elle avait été alors remontée et remise en place à l'intérieur d'un petit sanctuaire. L'examen d'une photo de cette réinstallation (fig. $5 \mathrm{~b}$ ) rend évident le rapprochement avec l'image récemment publiée. La similitude des proportions, du port et de la posture est plus que frappante; les impacts et des décollements de la pierre sur les oreilles et sur la trompe sont les mêmes. La statue monolithe, déjà fracturée en plusieurs morceaux, a manifestement été arrachée de sa base puis abondamment restaurée. Les lacunes du front, les cassures du torse, le coude droit, le diadème que l'on voit aujourd'hui, ne sont vraisemblablement que des reprises. Le pectoral est altéré, moins souple, plus plat, et sur cette zone, la pierre fait fortement penser à une surface artificielle. Ces observations seraient très aisément contrôlables: par des photographies rapprochées, prises sous le même angle; par la détection de broches, témoins des cassures et assemblages du Ganeśa du Prasat Bak; ou par des comparaisons d'empreintes avec l'arrachement de la base restée sur le site; ou encore par l'analyse des grès. Il est difficile, en tout état de cause, d'imaginer l'existence d'une image jumelle qui n'aurait jamais été repérée auparavant. Le Prasat Bak, est un petit sanctuaire dans lequel il n'y pas d'emplacement pour une autre statue que celle qui a été remontée par Parmentier et qui a disparu depuis. Tout laisse penser que le Ganeśa présenté dans le livre de E. Bunker et D. Latchford est bien celui qui a disparu du Prasat Bak pendant les années 80 ou au début des années 90 .

De tels exemples montrent combien il est nécessaire de prendre de la distance vis-à-vis de pièces dont la provenance n'est pas définie, et qui surgissent inopinément. Même si parfois un examen de surface incline à les authentifier, la documentation scientifique et la traçabilité de leur parcours sont essentiels, l'acuité de l'observation primordiale.

BERTRAND PORTE

Atelier du Musée national de Phnom Penh/FFEO

Notes

1 I:mma C. Bunker and Douglas Latchford, Adoration and Glory. The Golden Age of Khmer Art, Chicago, Art Media Resources, 2004 IKodansha Europe I.td. distr.], p. 70-71

2 Durga, Musée National de Phnom Penh, entrée dans la collection du musée en 1953. Inventaire: 5759. Catalogues: B819, B42.7, Ka1631. Provenance: Tuol Komnat (Char Ponhea lieng Kompong Trabeck, Province de Prey Veng). Style de Prei kmeing, fin vII"-début VIII"s.

3 Adoration and Glory..., op. cit., p. 168-170.

4 Fragments repérés et photographiés par Christophe Pottier (EFEO) en janvier 2004

5 Henri Parmentier, l'art khmer classique, Paris. EFEO, 1939, p. 74-75. "Chronique", Bulletin de l'licole française d'lixtrême-Orient, vol. XXX-1. 